\title{
On Inhomogeneity of a String Bit Model for Quantum Gravity
}

\author{
C.-W. H. Lee ${ }^{1}$ and R. B. Mann ${ }^{2}$ \\ Department of Physics, Faculty of Science, University of Waterloo, Waterloo, \\ Ontario, Canada, N2L $3 G 1$.
}

August 9, 2004

\begin{abstract}
We study quantum gravitational effect on a two-dimensional open universe with one particle by means of a string bit model. We find that matter is necessarily homogeneously distributed if the influence of the particle on the size of the universe is optimized.
\end{abstract}

PACS numbers: $04.60 . \mathrm{Kz}, 04.60 . \mathrm{Nc} .71 .10 . \mathrm{Fd}$.

Keywords: quantum gravity, string bit models, large- $N$ limit, scaling limit, Hubbard model.

${ }^{1}$ e-mail address: lee@scimail.uwaterloo.ca

2e-mail address: mann@avatar.uwaterloo.ca 


\section{Introduction}

As the quest for a quantum theory of gravity continues, two-dimensional theories continue to play an important role $[1,2,3,4,5,6]$. Their simplified setting affords considerably greater mathematical progress, offering the promise that the insights gleaned from this approach can be implemented in higher dimensions.

Discrete models of quantum gravity have been of increasing interest in this context in recent years. In two dimensions such models are called Hamiltonian string bit models, in which the spatial metric degree of freedom is discretized by introducing a distance cutoff $a_{*}>0$. The volume of spacetime is then $n a_{*}$ where $n$ is the (integer) number of links in a slice, with equal-time slices taken to be polygonal loops for a closed universe or straight lines with discrete segments for an open universe. Each slice is described by a pure quantum state $|n\rangle$ with $a_{*}$ fixed, and the set of such states forms an orthogonal basis for the Hilbert space of states. Adjacent links are created/destroyed by creation/annihiliation operators, preserving the locality of the model. The continuum limits of twodimensional Lorentzian models of quantum gravity can be obtained from these string bit Hamiltonian models [7].

It has recently been shown that string-bit models can be extended from the pure gravity case to include couplings to matter [8]. This is done by introducing new creation and annihilation operators that act to create and destroy particles that reside on the links. In this sense the particle is represented by a 'coloured link'. By homogeneously superposing the coloured link throughout the equaltime slice, a variety of interesting results can be obtained. Single-particle closed and open universe models were found to respectively behave like empty open and closed universes. A broad class of closed-universe models with indistinguishable bosons were shown to be special cases of the fractional sector model that plays the role of a metamodel for this class. The solution to the fractional sector model at the continuum limit was shown to have a transition amplitude that is exactly the same as the $s l(2)$ gravity model in Ref. [7].

In this paper we generalize the homogeneous model to include situations in which the particle is in an arbitrary superposition of locations on a collection of string bits. In other words, the matter distribution may be inhomogeneous on a time slice. We find that if certain parameters in the model that measure the effect of the particle on the size of a time-slice take on specific values, then inhomogeneity will not arise; the model is physically equivalent to homogenous single-particle models, having the same transition amplitude in the continuum limit.

The outline of our paper is as follows. In Section 2, we briefly review the general formalism of the string bit model and describe in detail the model we study in subsequent sections. In Sections 3 and 4, we work out the eigenstates of this model in asymptotic form. In Section 5, we classify the solutions. One class of solutions describes two empty open universes glued together; another class describes a universe with homogeneous matter distribution; yet another

class offers other possibilities. In Section 6, we summarize the key results of this article scattered throughout Sections 2 to 5 . 


\section{The model}

Consider an open two-dimensional universe with two boundaries. Considering it as a succession of time slices, we discretize each time slice into a number of links. The two outermost links represent the two boundaries, some interior links represent empty space, and other interior links represent matter; we will call them boundary links, empty links, and matter links, respectively. For the sake of simplicity, in this article we will consider only the case in which there is exactly one indestructible and indivisible particle in a time slice. Hence there are exactly one matter link, two boundary links and an arbitrary non-negative number of empty links. The matter link may drift as time goes by, and the number of empty links may change.

Mathematically, a link is represented by a matrix of creation operators. In particular, the bosonic creation operators $a_{\nu}^{\dagger \mu}$, where $\mu$ and $\nu$ are any positive integers between 1 and a positive integer $N$ inclusive, represent an empty link. In addition, the creation operators

$$
\bar{q}^{\dagger \mu}, q_{\mu}^{\dagger} \text {, and } b_{\nu}^{\dagger \mu}
$$

represent the left-boundary link, the right-boundary link, and the matter link, respectively. Because we consider a one-particle model, it does not matter whether these three kinds of creation operators are bosonic or fermionic ${ }^{3}$; for the sake of definiteness, we will take all these operators to be bosonic. The corresponding annihilation operators are

$$
a_{\mu}^{\nu}, \bar{q}_{\mu}, q^{\mu}, \text { and } b_{\mu}^{\nu},
$$

respectively. They satisfy the standard canonical commutation relations:

$$
\begin{aligned}
{\left[a_{\mu_{1}}^{\mu_{2}}, a_{\mu_{3}}^{\dagger \mu_{4}}\right] } & =\delta_{\mu_{1}}^{\mu_{4}} \delta_{\mu_{3}}^{\mu_{2}}, \\
{\left[b_{\mu_{1}}^{\mu_{2}}, b_{\mu_{3}}^{\mu_{4}}\right] } & =\delta_{\mu_{1}}^{\mu_{4}} \delta_{\mu_{3}}^{\mu_{2}}, \\
{\left[q^{\mu_{2}}, q_{\mu_{1}}^{\dagger}\right] } & =\delta_{\mu_{1}}^{\mu_{2}}, \text { and } \\
{\left[\bar{q}_{\mu_{1}}, \bar{q}^{\dagger \mu_{2}}\right] } & =\delta_{\mu_{1}}^{\mu_{2}} .
\end{aligned}
$$

All other commutators among these operators vanish.

The quantum state of a time slice with $m$ empty links to the left and $n$ empty links to the right of the matter link, where $m$ and $n$ are non-negative integers, is

$$
|m, n\rangle:=\frac{1}{N^{(m+n+2) / 2}} \bar{q}^{\dagger}\left(a^{\dagger}\right)^{m} b^{\dagger}\left(a^{\dagger}\right)^{n} q^{\dagger}|\Omega\rangle,
$$

where $|\Omega\rangle$ is the vacuum state and the matrix product $\bar{q}^{\dagger}\left(a^{\dagger}\right)^{m} b^{\dagger}\left(a^{\dagger}\right)^{n} q^{\dagger}$ is defined as

$$
\bar{q}^{\dagger}\left(a^{\dagger}\right)^{m} b^{\dagger}\left(a^{\dagger}\right)^{n} q^{\dagger}:=\bar{q}^{\dagger \mu_{1}} a_{\mu_{1}}^{\dagger \mu_{2}} a_{\mu_{2}}^{\dagger \mu_{3}} \cdots a_{\mu_{m}}^{\dagger \mu_{m+1}} b_{\mu_{m+1}}^{\dagger \nu_{1}} a_{\nu_{1}}^{\dagger \nu_{2}} a_{\nu_{2}}^{\dagger \nu_{3}} \cdots a_{\nu_{n}}^{\dagger \nu_{n+1}} q_{\nu_{n+1}}^{\dagger}
$$

\footnotetext{
${ }^{3}$ However whether they are bosonic or fermionic leads to different results in more complicated models.
} 
where we have employed the summation convention. In the large- $N$ limit, the norm of this state is 1 :

$$
\lim _{N \rightarrow \infty}\langle m, n \mid m, n\rangle=1
$$

The Hilbert space $\mathcal{H}$ of all quantum states of a time slice is spanned by all possible $|m, n\rangle$.

A suitable Hamiltonian for this one-particle open-universe model ${ }^{4}$ is:

$$
H:=H_{0}+K H_{K}+\lambda H_{-1}+\lambda H_{1}
$$

where

$$
\begin{aligned}
H_{0}:= & \operatorname{Tr} a^{\dagger} a+V \operatorname{Tr} b^{\dagger} b+\frac{1}{4} \bar{q}^{\dagger} \bar{q}+\frac{1}{4}\left(q^{\dagger}\right)^{t} q^{t} \\
H_{K}:= & -\frac{1}{N} \operatorname{Tr} a^{\dagger} b^{\dagger} a b-\frac{1}{N} \operatorname{Tr} b^{\dagger} a^{\dagger} b a-\frac{\gamma}{N} \bar{q}^{\dagger} b^{\dagger} b \bar{q}-\frac{\gamma}{N}\left(q^{\dagger}\right)^{t}\left(b^{\dagger}\right)^{t} b^{t} q^{t}, \\
H_{-1}:= & \frac{1}{\sqrt{N}} \operatorname{Tr}\left(a^{\dagger}\right)^{2} a+\frac{\xi}{\sqrt{N}}\left(\operatorname{Tr} b^{\dagger} a^{\dagger} b+\operatorname{Tr} a^{\dagger} b^{\dagger} b\right) \\
& +\frac{\eta}{N \sqrt{N}}\left(\bar{q}^{\dagger} b^{\dagger} a^{\dagger} b \bar{q}+\left(q^{\dagger}\right)^{t}\left(b^{\dagger}\right)^{t}\left(a^{\dagger}\right)^{t} b^{t} q^{t}\right)
\end{aligned}
$$

and

$$
\begin{aligned}
H_{1}: & =\frac{1}{\sqrt{N}} \operatorname{Tr} a^{\dagger} a^{2} \\
& +\frac{\xi}{\sqrt{N}}\left(\operatorname{Tr} b^{\dagger} a b+\operatorname{Tr} b^{\dagger} b a\right)+\frac{\eta}{N \sqrt{N}}\left(\bar{q}^{\dagger} b^{\dagger} a b \bar{q}+\left(q^{\dagger}\right)^{t}\left(b^{\dagger}\right)^{t} a^{t} b^{t} q^{t}\right) .
\end{aligned}
$$

The superscripts $t$ in Eqs.(1), (2), (3), and (4) denote the transposes of the matrices concerned.

Let us describe the action of $H$ on $\mathcal{H}$ in the large- $N$ limit. (See ref. [9] for a review of how any operator that is the trace of a product of matrices of creation or annihilation operators introduced above behaves in the large- $N$ limit.) In $H_{0}$, the first term counts the number of empty links in a time-slice, thereby measuring the energy of empty space, which is proportional to its size. The second term may be interpreted as a mass term for the matter with $V$ being the mass. The third and fourth terms are the energy terms of the left and right boundaries, respectively. $H_{K}$ is a variant of the Hubbard model in the context of the string bit model [10]. The action of the first two terms of $H_{K}$ on $|m, n\rangle$ causes the matter link to respectively drift to the right or left. The third and fourth terms may be thought of as open boundary conditions for this Hubbard model. $\gamma$ is a constant. If $\gamma=1$, then $H_{K}$ leaves invariant the subspace of $\mathcal{H}$

\footnotetext{
${ }^{4}$ The differences between this Hamiltonian and the Hamiltonian of a similar model in Ref. [8] are:

1. the coefficients of $\bar{q}^{\dagger} \bar{q}$ and $\left(q^{\dagger}\right)^{t} q^{t}$ are $1 / 4$ instead of $-1 / 4$, and

2. $\gamma=1$ in the Hamiltonian in Ref. [8].
} 
consisting of all quantum states of time slices in which matter is homogeneously distributed [8]. The coefficient $K$ in $H$ may be interpreted as the Hubbard constant; the larger its value, the easier the matter link drifts.

$H_{-1}$ is a collection of terms whose action increases the number of links of a discretised time slice by one. The first term causes an empty link to split into two. The terms proportional to $\xi$, a constant, cause the matter link to split into itself and an empty link; the new empty link may reside on the left or right of the matter link. The terms proportional to $\eta$, another constant, also cause the matter link to split into itself and an empty link if the matter link is adjacent to a boundary link; the new empty link must be on the interior side of the matter link. $H_{1}$ is the Hermitian conjugate of $H_{-1}$. The action of any term of $H_{1}$ causes two adjacent links to combine together. The constant coefficient $\lambda$ measures the ease with which a link splits into two or the ease with which adjacent links combine together.

We see from the above description that $H$ is the simplest non-trivial Hamiltonian that allows for a variation of the size of the time slice and the matter link to move around as time goes by. Furthermore, the matter link may interact with the boundary links in a special manner as displayed by the boundary terms.

\section{An Asymptotic Expression for Eigenstates}

We consider now general superpositions of states $|m, n\rangle$, where

$$
\phi:=\sum_{m, n=0}^{\infty} a_{m n}|m, n\rangle
$$

is an eigenstate of $H$ and $E$ the corresponding eigenenergy. If any two coefficients of quantum states with the same total number of links are equal, i.e. if

$$
a_{m+k, n-k}=a_{m n}
$$

for any non-negative values of $m$ and $n$ and any negative value of $k$ such that $k \leq n$, then matter is homogeneously distributed. The action of the Hamiltonian on $\phi$ yields

$$
\begin{aligned}
\left(m+n+\frac{1}{2}+V\right) a_{m n} \\
\quad-K a_{m+1, n-1}-K a_{m-1, n+1}+\lambda(m-1+\xi) a_{m-1, n}+\lambda(n-1+\xi) a_{m, n-1} \\
\quad+\lambda(m+\xi) a_{m+1, n}+\lambda(n+\xi) a_{m, n+1}=E a_{m n}
\end{aligned}
$$

for any positive integers $m$ and $n$,

$$
\begin{aligned}
& \left(n+\frac{1}{2}+V-\gamma K\right) a_{0 n}-K a_{1, n-1} \\
& \quad+\lambda(n-1+\xi+\eta) a_{0, n-1}+\xi \lambda a_{1 n}+\lambda(n+\xi+\eta) a_{0, n+1}=E a_{0 n}
\end{aligned}
$$


for any positive integer $n$,

$$
\begin{aligned}
(m+ & \left.\frac{1}{2}+V-\gamma K\right) a_{m 0}-K a_{m-1,1} \\
& +\lambda(m-1+\xi+\eta) a_{m-1,0}+\xi \lambda a_{m 1}+\lambda(m+\xi+\eta) a_{m+1,0}=E a_{m 0}
\end{aligned}
$$

for any positive integer $m$, and

$$
\left(\frac{1}{2}+V-2 \gamma K\right) a_{00}+\lambda(\xi+\eta)\left(a_{10}+a_{01}\right)=E a_{00} .
$$

It follows from Eq. (6) that

$$
m\left(\lambda a_{m+1, n}+a_{m n}+\lambda a_{m-1, n}\right)+n\left(\lambda a_{m, n+1}+a_{m n}+\lambda a_{m, n-1}\right) \simeq 0
$$

if both $m$ and $n$ are large. Hence

$$
a_{m n} \sim p^{m+n},
$$

where $p$ is the solution to the quadratic equation

$$
\lambda p^{2}+p+\lambda=0 .
$$

It is thus natural to write $a_{m n}$ as

$$
a_{m n}:=b_{m n} p^{m+n}
$$

for any non-negative integers $m$ and $n$. Normalizability implies that $b_{m n}$ increases with $m$ and $n$ polynomially. Since we are only interested in the continuum limit at which

$$
\lambda=-\frac{1}{2} \exp \left(-\frac{\Lambda a_{*}^{2}}{2}\right),
$$

where the parameter $a_{*}$ tends to 0 , and the number of string bits in a time slice is inversely proportional to $a_{*}$, we may try the ansatz

$$
b_{m n}:=\sum_{r=0}^{\infty} \sum_{s=0}^{\infty} c_{r s} a_{*}^{r+s} m^{r} n^{s}+\mathcal{O}\left(a_{*}\right),
$$

where both $m$ and $n$ are of order $1 / a_{*}$ so that $a_{*}^{r+s} m^{r} n^{s}$ is of order unity for any values of $r$ and $s$. The $c_{r s}$ are constants and only a finite number of them are non-zero. Higher order terms in $b_{m n}$ may be written as

$$
\sum_{i=1}^{\infty} \sum_{r=0}^{\infty} \sum_{s=0}^{\infty} c_{r s}^{(i)} a_{*}^{r+s+i} m^{r} n^{s}
$$

where $c_{r s}^{(i)}$ are constants. 
Upon substitution of Eqs. (11) and (12) into Eq. (6) we find

$$
\begin{aligned}
\lambda(m+ & +\xi) p^{2} \sum_{r=0}^{\infty} \sum_{s=0}^{\infty} c_{r s} a_{*}^{r+s}(m+1)^{r} n^{s} \\
& +\lambda(n+\xi) p^{2} \sum_{r=0}^{\infty} \sum_{s=0}^{\infty} c_{r s} a_{*}^{r+s} m^{r}(n+1)^{s} \\
& +\left(m+n+\frac{1}{2}+V-E\right) p \sum_{r=0}^{\infty} \sum_{s=0}^{\infty} c_{r s} a_{*}^{r+s} m^{r} n^{s} \\
& -K p \sum_{r=0}^{\infty} \sum_{s=0}^{\infty} c_{r s} a_{*}^{r+s}(m+1)^{r}(n-1)^{s} \\
& -K p \sum_{r=0}^{\infty} \sum_{s=0}^{\infty} c_{r s} a_{*}^{r+s}(m-1)^{r}(n+1)^{s} \\
& +\lambda(m-1+\xi) \sum_{r=0}^{\infty} \sum_{s=0}^{\infty} c_{r s} a_{*}^{r+s}(m-1)^{r} n^{s} \\
& +\lambda(n-1+\xi) \sum_{s=0}^{\infty} \sum_{s=0}^{\infty} c_{r s} a_{*}^{r+s} m^{r}(n-1)^{s} \simeq 0 .
\end{aligned}
$$

Using Eq. (10) to simplify Eq. (13) reveal that there are no terms of order $1 / a_{*}$ on the left hand side of Eq. (13). It also follows from Eq. (10) that terms neglected in the ansatz (12) contribute to terms of order $a_{*}$ or higher on the left hand side of Eq. (13).

Assume that the continuum limit is well defined. Then the eigenenergy $E$ is order $a_{*}$. Let us introduce the following Maclaurin series expansions in $a_{*}$ :

$$
\begin{aligned}
V & :=V_{0}+V_{1} \sqrt{\Lambda} a_{*}+\mathcal{O}\left(a_{*}^{2}\right), \\
K & :=K_{0}+K_{1} \sqrt{\Lambda} a_{*}+\mathcal{O}\left(a_{*}^{2}\right), \\
\gamma & :=\gamma_{0}+\gamma_{1} \sqrt{\Lambda} a_{*}+\mathcal{O}\left(a_{*}^{2}\right), \\
\xi & :=\xi_{0}+\xi_{1} \sqrt{\Lambda} a_{*}+\mathcal{O}\left(a_{*}^{2}\right), \text { and } \\
\eta & :=\eta_{0}+\eta_{1} \sqrt{\Lambda} a_{*}+\mathcal{O}\left(a_{*}^{2}\right) .
\end{aligned}
$$

After some algebra, we find the sum of the terms of order unity on the left hand side of Eq. (13) to be

$$
\sum_{r=0}^{R} \sum_{s=0}^{S} c_{r s} a_{*}^{r+s} m^{r} n^{s}\left(\frac{3}{2}+V_{0}-2 K_{0}-2 \xi_{0}\right) .
$$

Hence

$$
\frac{3}{2}+V_{0}-2 K_{0}-2 \xi_{0}=0
$$


is a necessary condition for the existence of the continuum limit. It follows from Eqs. (14) and (10) that terms neglected in the ansatz (12) contribute to terms of order $a_{*}^{2}$ or higher on the left hand side of Eq. (13).

The sum of the terms of order $a_{*}$ on the left hand side of Eq. (13) is

$$
\begin{aligned}
& -\frac{1}{2} \sum_{r=0}^{\infty} \sum_{s=0}^{\infty} c_{r+1, s} a_{*}^{r+s+1} m^{r} n^{s}(r+1)^{2} \\
& -\frac{1}{2} \sum_{r=0}^{\infty} \sum_{s=0}^{\infty} c_{r, s+1} a_{*}^{r+s+1} m^{r} n^{s}(s+1)^{2} \\
& +\sum_{r=0}^{\infty} \sum_{s=0}^{\infty} c_{r s} a_{*}^{r+s} m^{r} n^{s}\left(r+s+V_{1}-2 K_{1}-2 \xi_{1}+1-\frac{E}{\sqrt{\Lambda} a_{*}}\right) \sqrt{\Lambda} a_{*} .
\end{aligned}
$$

Hence

$$
\begin{gathered}
c_{r s} \sqrt{\Lambda}\left(r+s+V_{1}-2 K_{1}-2 \xi_{1}+1-\frac{E}{\sqrt{\Lambda} a_{*}}\right) \\
-\frac{1}{2} c_{r+1, s}(r+1)^{2}-\frac{1}{2} c_{r, s+1}(s+1)^{2}=0
\end{gathered}
$$

for all non-negative integer values of $r$ and $s$.

We solve this relation by assuming that the series in $c_{r s}$ truncates. Let $R_{0}$ and $S_{0}$ be non-negative integers such that $c_{R_{0} S_{0}} \neq 0$ but $c_{R_{0}+r^{\prime}, S_{0}+s^{\prime}}=0$ for any non-negative integers $r^{\prime}$ and $s^{\prime}$ such that $r^{\prime}+s^{\prime}>0$. Then Eq. (15) implies that

$$
E=(1+Q+\tilde{h}) \sqrt{\Lambda} a_{*},
$$

where $Q=R_{0}+S_{0}$ and $\tilde{h}=V_{1}-2 K_{1}-2 \xi_{1}$. Thus if $R^{\prime}$ and $S^{\prime}$ are non-negative integers such that $c_{R^{\prime} S^{\prime}} \neq 0$ but $c_{R^{\prime}+r^{\prime}, S^{\prime}+s^{\prime}}=0$ for any non-negative integers $r^{\prime}$ and $s^{\prime}$ such that $r^{\prime}+s^{\prime}>0$, then $R^{\prime}+S^{\prime}=R_{0}+S_{0}$. One may then readily verify that

$$
c_{r s}=\sum_{R=0}^{Q}(-2 \sqrt{\Lambda})^{r+s-Q} \frac{R !(Q-R) !}{r ! s !}\left(\begin{array}{c}
R \\
r
\end{array}\right)\left(\begin{array}{c}
Q-R \\
s
\end{array}\right) c_{R, Q-R},
$$

is the unique solution to Eq. (15). Combining Eqs. (5), (11), (12) and (17) yields an asymptotic expression for any eigenstate. We will determine the constraints on the values of $Q$ and $c_{R, Q-R}$ in the next section.

\section{Boundary Conditions}

Let us analyze Eqs. (7), (8), and (9) in this section. Let

$$
f_{Q, R}:=\frac{R !(Q-R) !}{(-2 \sqrt{\Lambda})^{Q}} c_{Q-R, R}
$$


It then follows from Eqs. (12) and (17) that

$$
b_{m n} \simeq \sum_{S=0}^{Q} f_{Q S} \sum_{r=0}^{Q-S} \sum_{s=0}^{S}\left(\begin{array}{c}
Q-S \\
r
\end{array}\right)\left(\begin{array}{c}
S \\
s
\end{array}\right) \frac{\left(-2 \sqrt{\Lambda} a_{*} m\right)^{r}\left(-2 \sqrt{\Lambda} a_{*} n\right)^{s}}{r ! s !} .
$$

Substituting Eqs. (11) and (18) into Eq. (7) yields

$$
\begin{aligned}
(n+ & \left.\frac{1}{2}+V-\gamma K-E\right) p \sum_{S=0}^{Q} f_{Q S} \sum_{s=0}^{S} \frac{\left(-2 \sqrt{\Lambda} a_{*}\right)^{s}}{s !}\left(\begin{array}{c}
S \\
s
\end{array}\right) n^{s} \\
& -K p \sum_{S=0}^{Q} f_{Q S} \sum_{r=0}^{Q-S} \sum_{s=0}^{S} \frac{\left(-2 \sqrt{\Lambda} a_{*}\right)^{r+s}}{r ! s !}\left(\begin{array}{c}
Q-S \\
r
\end{array}\right)\left(\begin{array}{c}
S \\
s
\end{array}\right)(n-1)^{s} \\
& +\lambda(n-1+\xi+\eta) \sum_{S=0}^{Q} f_{Q S} \sum_{s=0}^{S} \frac{\left(-2 \sqrt{\Lambda} a_{*}\right)^{s}}{s !}\left(\begin{array}{c}
S \\
s
\end{array}\right)(n-1)^{s} \\
& +\lambda \xi p^{2} \sum_{S=0}^{Q} f_{Q S} \sum_{r=0}^{Q-S} \sum_{s=0}^{S} \frac{\left(-2 \sqrt{\Lambda} a_{*}\right)^{r+s}}{r ! s !}\left(\begin{array}{c}
Q-S \\
r
\end{array}\right)\left(\begin{array}{c}
S \\
s
\end{array}\right) n^{s} \\
& +\lambda(n+\xi+\eta) p^{2} \sum_{S=0}^{Q} f_{Q S} \sum_{s=0}^{S} \frac{\left(-2 \sqrt{\Lambda} a_{*}\right)^{s}}{s !}\left(\begin{array}{c}
S \\
s
\end{array}\right)(n+1)^{s} \simeq 0
\end{aligned}
$$

Once again, we can use Eq. (10) to show that the sum of the terms of order $1 / a_{*}$ on the left hand side of Eq. (19) vanishes and that terms neglected in the ansatz (12) contribute to terms of order $a_{*}$ or higher. The sum of the terms of order unity is

$$
\sum_{S=0}^{Q} f_{Q S} \sum_{s=0}^{S}\left(\begin{array}{c}
S \\
s
\end{array}\right) \frac{\left(-2 \sqrt{\Lambda} a_{*} n\right)^{s}}{s !}\left[\left(1-\gamma_{0}\right) K_{0}+\frac{1}{2} \xi_{0}-\eta_{0}-\frac{1}{2}\right] .
$$

where we used Eq. (14) and the various Maclaurin series. Hence

$$
\left(1-\gamma_{0}\right) K_{0}+\frac{1}{2} \xi_{0}-\eta_{0}-\frac{1}{2}=0
$$

is another necessary condition for the continuum limit to be well defined. It follows from Eqs. (20) and (10) that terms neglected in the ansatz (12) contribute to terms of order $a_{*}^{2}$ or higher on the left hand side of Eq. (19).

The sum of the terms of order $a_{*}$ on the left hand side of Eq. (19) is

$$
\begin{aligned}
\sum_{S=0}^{Q} f_{Q S} \sum_{s=0}^{S}\left(\begin{array}{c}
S \\
s
\end{array}\right) \frac{\left(-2 \sqrt{\Lambda} a_{*} n\right)^{s}}{s !} \sqrt{\Lambda} a_{*}\left[\left(\xi_{0}+2 K_{0}-1\right)(Q-S)\right. \\
\left.\quad-2 K_{0} \frac{S-s}{s+1}+\left(-1+\gamma_{0}+\gamma_{1}\right) K_{0}+\left(1-\gamma_{0}\right) K_{1}-\frac{7}{2} \xi_{1}+\eta_{0}-\eta_{1}\right] .
\end{aligned}
$$


(We have made use of Eq. (14) to obtain the above formula.) Since $n$ is arbitrary, we obtain the constraint

$$
\sum_{S=s}^{Q} f_{Q S}\left(\begin{array}{c}
S \\
s
\end{array}\right)\left[\left(\xi_{0}-1\right)(Q-S)+2 K_{0}\left(Q-S \frac{s+2}{s+1}+\frac{s}{s+1}\right)+C\right]=0,
$$

where

$$
C:=\left(-1+\gamma_{0}+\gamma_{1}\right) K_{0}+\left(1-\gamma_{0}\right) K_{1}-\frac{7}{2} \xi_{1}+\eta_{0}-\eta_{1}
$$

is a constant and $s$ is any non-negative integer.

Repeating the above argument for Eq. (8) leads to the same necessary Constraint (20) and the constraint

$$
\begin{aligned}
& \sum_{R=r}^{Q} f_{Q, Q-R}\left(\begin{array}{c}
R \\
r
\end{array}\right)\left[\left(\xi_{0}-1\right)(Q-R)\right. \\
& \left.\quad+2 K_{0}\left(Q-R \frac{r+2}{r+1}+\frac{r}{r+1}\right)+C\right]=0
\end{aligned}
$$

for any non-negative value of $r$.

Let us turn our attention to Eq. (9). Substituting Eqs. (11) and (18) into Eq. (9) and using (14) and (20), we get

$$
\begin{aligned}
& \eta_{0} \sum_{S=0}^{Q} f_{Q S}+\sqrt{\Lambda} a_{*}\left[\left(\xi_{0}+\eta_{0}-1\right)(Q+1)\right. \\
& \left.\quad+2\left(1-\gamma_{0}\right) K_{1}-2 \gamma_{1} K_{0}+\xi_{1}-\eta_{1}\right] \sum_{S=0}^{Q} f_{Q S}+\rho\left(a_{*}\right)=0
\end{aligned}
$$

where $\rho\left(a_{*}\right)$ comes from terms neglected in the ansatz (12). It is of order $a_{*}$ or higher and is directly proportional to $\eta_{0}$. As a result,

$$
\eta_{0} \sum_{S=0}^{Q} f_{Q S}=0
$$

and

$$
\begin{aligned}
& {\left[\left(\xi_{0}+\eta_{0}-1\right)(Q+1)\right.} \\
& \left.\quad+2\left(1-\gamma_{0}\right) K_{1}-2 \gamma_{1} K_{0}+\xi_{1}-\eta_{1}\right] \sum_{S=0}^{Q} f_{Q S}+\frac{\rho\left(a_{*}\right)}{\sqrt{\Lambda} a_{*}}=0
\end{aligned}
$$

are two additional necessary conditions for the continuum limit to be well defined.

\section{Different Scenarios}

Let us solve the constraints and classify the solutions. 


\subsection{Direct Product of Empty Open Universes}

One solution to Constraints (23) and (24) is $\eta_{0}=0$ (which in turn implies that $\left.\rho\left(a_{*}\right)=0\right), \xi_{0}=1$, and

$$
2\left(1-\gamma_{0}\right) K_{1}-2 \gamma_{1} K_{0}+\xi_{1}-\eta_{1}=0 .
$$

Suppose that the matter link cannot drift at the continuum limit, i.e.

$$
K_{0}=0 .
$$

Then Constraints (21) and (22) lead to

$$
C=0,
$$

and the ratios $f_{Q 0}: f_{Q 1}: \cdots: f_{Q Q}$ is arbitrary. Furthermore, Eq. (14) implies that $V_{0}=1 / 2$. Comparison of this value of $V_{0}$ with the coefficients of the boundary terms in Eq. (1) reveals that the matter link is nothing but the connection of a right and a left boundary link. Moreover, comparison of the energy spectra of this one-particle model (16) and the $s l(2)$ gravity model with $h=1 / 2$ (Eq. (30) of Ref. $[7])^{5}$, and comparison of the asymptotic expressions of the eigenstates of this model (Eqs. (5), (11), and (18)) and of the $s l(2)$ gravity model as partly displayed in Eq. (31) of Ref. [7] confirm that this model is equivalent to two empty open universes joined together at one of their ends.

The transition amplitude at the continuum limit is defined as

$$
\tilde{G}\left(L_{1}, L_{2} ; L_{1}^{\prime}, L_{2}^{\prime} ; T\right):=\lim _{a_{*} \rightarrow 0} \frac{1}{a_{*}^{2}}\left\langle\frac{L_{1}^{\prime}}{a_{*}}, \frac{L_{2}^{\prime}}{a_{*}}\left|e^{-t H}\right| \frac{L_{1}}{a_{*}}, \frac{L_{2}}{a_{*}}\right\rangle .
$$

It follows immediately from the argument in the preceding paragraph and Eq. (38) of Ref. [7] that

$$
\begin{aligned}
& \tilde{G}\left(L_{1}, L_{2} ; L_{1}^{\prime}, L_{2}^{\prime} ; T\right)=\frac{\Lambda e^{-2 \tilde{h} \sqrt{\Lambda} T}}{\sinh ^{2}(\sqrt{\Lambda} T)} \\
& \quad \cdot e^{-\sqrt{\Lambda}\left(L_{1}+L_{2}+L_{1}^{\prime}+L_{2}^{\prime}\right) \operatorname{coth}(\sqrt{\Lambda} T)} I_{0}\left(\frac{2 \sqrt{\Lambda L_{1} L_{1}^{\prime}}}{\sinh (\sqrt{\Lambda} T)}\right) I_{0}\left(\frac{2 \sqrt{\Lambda L_{2} L_{2}^{\prime}}}{\sinh (\sqrt{\Lambda} T)}\right) \cdot(27)
\end{aligned}
$$

\subsection{Homogeneous Matter Distribution}

Consider again $\eta_{0}=0, \xi_{0}=1$, and $2\left(1-\gamma_{0}\right) K_{1}-2 \gamma_{1} K_{0}+\xi_{1}-\eta_{1}=0$ (Eq. (25)). Assume, however, that $K_{0} \neq 0$ so that the matter link may drift. We will show by contradiction that

\section{Theorem 5.1}

$$
C=0
$$

\footnotetext{
${ }^{5}$ Note that $\tilde{h}=0$ in Ref. [7].
} 
Proof. Suppose, on the contrary, that $C \neq 0$. Then Eq. (21) with $s=Q$ implies that

$$
f_{Q Q}=0 .
$$

Similarly, Eq. (22) with $r=Q$ implies that

$$
f_{Q 0}=0 .
$$

Treat Eq. (21) with $s=1,2, \ldots, Q-1$ as a set of simultaneous linear equations in $f_{Q 1}, f_{Q 2}, \ldots$, and $f_{Q, Q-1}$. A non-trivial solution exists only if the determinant of a matrix of coefficients vanishes, i.e.

$$
\prod_{s=1}^{Q-1}\left(\begin{array}{l}
s \\
s
\end{array}\right)\left[2 K_{0}\left(Q-s \frac{s+2}{s+1}+\frac{s}{s+1}\right)+C\right]=0 .
$$

Hence there exists a positive integer $s_{0}<Q$ such that

$$
2 K_{0}\left(Q-s_{0} \frac{s_{0}+2}{s_{0}+1}+\frac{s_{0}}{s_{0}+1}\right)+C=0 .
$$

Thus

$$
C=-2 K_{0}\left(Q-s_{0}\right) .
$$

It then follows from Eq. (21) with $s=Q-1, Q-2, \ldots$, and $s_{0}+1$ that

$$
f_{Q, Q-1}=f_{Q, Q-2}=\cdots=f_{Q, s_{0}+1}=0
$$

and from the same equation that

$$
\sum_{S=s}^{s_{0}} f_{Q s}\left(\begin{array}{c}
S \\
s
\end{array}\right)\left(s_{0}-S \frac{s+2}{s+1}+\frac{s}{s+1}\right)=0
$$

for $s=0,1, \ldots$, and $s_{0}-1$. Theorem A.1 in Appendix A with $Q^{\prime}=s_{0}$ and Eq. (28) then imply that

$$
f_{Q, s_{0}}=f_{Q, s_{0}-1}=\cdots=f_{Q 0}=0 .
$$

However, this contradicts the assumption that there is a non-trivial solution among $f_{Q 1}, f_{Q 2}, \ldots$, and $f_{Q, Q-1}$. Q.E.D.

It follows from the above theorem and Eq. (21) that $f_{Q 0}, f_{Q 1}, \ldots$, and $f_{Q Q}$ must satisfy

$$
\sum_{S=s}^{Q} f_{Q S}\left(\begin{array}{c}
S \\
s
\end{array}\right)\left(Q-S \frac{s+2}{s+1}+\frac{s}{s+1}\right)=0
$$

for any non-negative value of $s$. Consequently, Theorem A.1 in Appendix A with $Q^{\prime}=Q$ implies that

$$
f_{Q 0}=f_{Q 1}=\cdots=f_{Q Q}
$$


is the only solution to Eqs. (21) and (22) if $\xi_{0}=1$. Then Eq. (18) implies that

$$
\begin{aligned}
b_{m n} & =f_{Q 0} \sum_{r=0}^{Q} \sum_{s=0}^{Q-r} \frac{\left(-2 \sqrt{\Lambda} a_{*} m\right)^{r}\left(-2 \sqrt{\Lambda} a_{*} n\right)^{s}}{r ! s !} \sum_{R=0}^{Q}\left(\begin{array}{c}
R \\
r
\end{array}\right)\left(\begin{array}{c}
Q-R \\
s
\end{array}\right) \\
& =f_{Q 0} \sum_{r=0}^{Q} \sum_{s=0}^{Q-r} \frac{\left(-2 \sqrt{\Lambda} a_{*} m\right)^{r}\left(-2 \sqrt{\Lambda} a_{*} n\right)^{s}}{r ! s !}\left(\begin{array}{c}
Q+1 \\
r+s+1
\end{array}\right) \\
& =f_{Q 0} \sum_{r=0}^{Q} \sum_{s=r}^{Q} \frac{\left(-2 \sqrt{\Lambda} a_{*} m\right)^{r}\left(-2 \sqrt{\Lambda} a_{*} n\right)^{s-r}(Q+1) !}{r !(s-r) !(s+1) !(Q-s) !} \\
& =f_{Q 0}(Q+1) \sum_{s=0}^{Q} \sum_{r=0}^{s} \frac{\left(-2 \sqrt{\Lambda} a_{*} m\right)^{r}\left(-2 \sqrt{\Lambda} a_{*} n\right)^{s-r}}{(s+1) !}\left(\begin{array}{c}
Q \\
s
\end{array}\right)\left(\begin{array}{c}
s \\
r
\end{array}\right) \\
& =f_{Q 0}(Q+1) \sum_{s=0}^{Q}\left(\begin{array}{c}
Q \\
s
\end{array}\right) \frac{\left(-2 \sqrt{\Lambda} a_{*}\right)^{s}(m+n)^{s}}{(s+1) !}
\end{aligned}
$$

where the second line follows from a standard combinatoric formula, which may be easily proved by induction on $Q$. Thus $b_{m n}$ and hence $a_{m n}$ depends on $m$ and $n$ via $m+n$. Physically, this means that on a time slice in any eigenstate, the matter link may be found anywhere with the same probability; matter is necessarily homogeneously distributed. It follows from Subsection 5.2 of Ref. [7], in particular Eq. (33) of the reference, that

$$
f_{Q 0}(Q+1)=2 \sqrt{(Q+1) \Lambda} a_{*} .
$$

Substitution of Eqs. (5), (11), (30), (31), and (16) into Definition (26) for the transition amplitude yields

$$
\begin{aligned}
& \tilde{G}\left(L_{1}, L_{2} ; L_{1}^{\prime}, L_{2}^{\prime} ; T\right)=4 \Lambda \sum_{R=1}^{\infty}(R+1) \\
& \quad \cdot \sum_{r=0}^{R} \sum_{s=0}^{R} \frac{(-2 \sqrt{\Lambda})^{r+s}\left(L_{1}+L_{2}\right)^{r}\left(L_{1}^{\prime}+L_{2}^{\prime}\right)^{s}}{(r+1) !(s+1) !} \\
& \quad \cdot\left(\begin{array}{c}
R \\
r
\end{array}\right)\left(\begin{array}{c}
R \\
s
\end{array}\right) e^{-\sqrt{\Lambda}\left(L_{1}+L_{2}+L_{1}^{\prime}+L_{2}^{\prime}\right)} e^{-2(1+\tilde{h}+R) \sqrt{\Lambda} T} .
\end{aligned}
$$

This formula differs from Eq. (36) of Ref. [7] in which $L=L_{1}+L_{2}, L^{\prime}=L_{1}^{\prime}+L_{2}^{\prime}$, and $h=1$ by a factor of

$$
\frac{e^{-2 \tilde{h} \sqrt{\Lambda} T}}{L L^{\prime}}
$$

only. Hence the subsequent calculation is parallel to the one in Ref. [7], and we may write down the final expression immediately:

$$
\tilde{G}\left(L_{1}, L_{2} ; L_{1}^{\prime}, L_{2}^{\prime} ; T\right)=\sqrt{\frac{\Lambda}{\left(L_{1}+L_{2}\right)\left(L_{1}^{\prime}+L_{2}^{\prime}\right)}} \frac{e^{-2 \tilde{h} \sqrt{\Lambda} T}}{\sinh (\sqrt{\Lambda} T)}
$$




$$
\cdot e^{-\sqrt{\Lambda}\left(L_{1}+L_{2}+L_{1}^{\prime}+L_{2}^{\prime}\right) \operatorname{coth}(\sqrt{\Lambda} T)} I_{1}\left(\frac{2 \sqrt{\Lambda\left(L_{1}+L_{2}\right)\left(L_{1}^{\prime}+L_{2}^{\prime}\right)}}{\sinh (\sqrt{\Lambda} T)}\right) .
$$

\subsection{Other Possilibities}

If $\eta_{0}=0$ but $\xi_{0} \neq 1$ or Constraint (25) does not hold, then Constraint (24) implies that

$$
\sum_{S=0}^{Q} f_{Q S}=0
$$

for all but perhaps one value of $Q$. If $\eta_{0} \neq 0$, then Constraint (23) implies that

$$
\sum_{S=0}^{Q} f_{Q S}=0
$$

for all values of $Q$. Furthermore, any solution, if it exists, has to satisfy Constraints (21) and (22). Working out solutions that satisfy all of these constraints remains an interesting research problem.

\section{Conclusion}

In the context of string bit models, quantum gravitational effects furnish considerable constraints on matter distributions. Indeed, the continuum limit for a string bit model with a single particle of matter is well defined only if the constraints

$$
\frac{3}{2}+V_{0}-2 K_{0}-2 \xi_{0}=0
$$

(Eq. (14)),

$$
\left(1-\gamma_{0}\right) K_{0}+\frac{1}{2} \xi_{0}-\eta_{0}-\frac{1}{2}=0,
$$

(Eq. (20)), Eq. (21), Eq. (22) and Eq. (24) are satisfied.

If $\eta=\mathcal{O}\left(a_{*}\right), \xi=1+\mathcal{O}\left(a_{*}\right)$, and Constraint (25) holds, we may rewrite Eqs. (14) and (20) as $V-2 K-1 / 2=\mathcal{O}\left(a_{*}\right)$ and $(1-\gamma) K=\mathcal{O}\left(a_{*}\right)$, respectively. In addition, if $K=\mathcal{O}\left(a_{*}\right)$, then the model describes two empty open universes glued together at one of their ends, and the transition amplitude is given by Eq. (27); otherwise, matter is necessarily homogeneously distributed, and the transition amplitude is given by Eq. (32).

According to Ref. [8], if we restrict ourselves to the case in which the actions of $H_{0}+K H_{K}, H_{-1}$, and $H_{1}$ are invariant on the Hilbert space individually, then $\gamma=1+\mathcal{O}\left(a_{*}\right), \xi=1+\mathcal{O}\left(a_{*}\right)$, and $\eta=\mathcal{O}\left(a_{*}\right)$. These are consistent with the findings of the previous paragraph. We also found that $V-2 K=$ $3 / 2+\mathcal{O}\left(a_{*}\right)$. At first glance, this violates Constraint (14); however, this apparent inconsistency would disappear if the coefficients of $\bar{q}^{\dagger} \bar{q}$ and $\left(q^{\dagger}\right)^{t} q^{t}$ in $H$ were chosen to be $1 / 4$ instead of $-1 / 4$ as in Ref. [8] (c.f. Eq. (1)). This confirms that 
the model studied in Section 3 of Ref. [8] is indeed just a special case of the model we are studying in this article.

The case $\eta \neq \mathcal{O}\left(a_{*}\right), \xi \neq 1+\mathcal{O}\left(a_{*}\right)$, or Constraint (25) does not hold offers other possibilities. Exploration of these possibilities remains an interesting project for the future.

\section{Acknowledgment}

We thank J. Ambjørn for discussion. This work was supported in part by the Natural Sciences and Engineering Research Council of Canada.

\section{Appendix}

\section{A A Combinatoric Formula}

Lemma A.1

$$
\sum_{S=s}^{Q^{\prime}}\left(\begin{array}{c}
S \\
s
\end{array}\right)\left(Q^{\prime}-S \frac{s+2}{s+1}+\frac{s}{s+1}\right)=0
$$

for any non-negative value of $s$ and any integer value of $Q^{\prime}$ such that $Q^{\prime} \geq s$.

Proof. For a fixed value of $s$, by an inductive argument on $Q^{\prime}$, one may readily show that

$$
\sum_{n=0}^{N-s}\left(\begin{array}{c}
n+s \\
n
\end{array}\right)=\left(\begin{array}{c}
N+1 \\
s+1
\end{array}\right) .
$$

It is straightforward to show that Eq. (33) holds true for $Q^{\prime}=s$. Assume that Eq. (33) holds true for $Q^{\prime}=Q_{0}$ for some integer $Q_{0}$ not less than $s$. Then

$$
\begin{aligned}
& \sum_{S=s}^{Q_{0}+1}\left(\begin{array}{c}
S \\
s
\end{array}\right)\left(Q_{0}+1-S \frac{s+2}{s+1}+\frac{s}{s+1}\right) \\
= & \sum_{S=s}^{Q_{0}}\left(\begin{array}{c}
S \\
s
\end{array}\right)+\left(\begin{array}{c}
Q_{0}+1 \\
s
\end{array}\right)\left[Q_{0}+1-\left(Q_{0}+1\right) \frac{s+2}{s+1}+\frac{s}{s+1}\right] \\
= & \sum_{n=0}^{Q_{0}-s}\left(\begin{array}{c}
n+s \\
n
\end{array}\right)-\left(\begin{array}{c}
Q_{0}+1 \\
s+1
\end{array}\right) \\
= & 0,
\end{aligned}
$$

where the second line follows from the inductive hypothesis and the last line follows from Eq. (34). Hence Eq.(33) holds true for $Q^{\prime}=Q_{0}+1$ as well. By mathematical induction, Eq. (33) holds true for all integer values of $Q^{\prime}$ such that $Q^{\prime} \geq s$. Q.E.D. 
Theorem A.1 If

$$
\sum_{S=s}^{Q^{\prime}} f_{Q^{\prime} S}\left(\begin{array}{c}
S \\
s
\end{array}\right)\left(Q^{\prime}-S \frac{s+2}{s+1}+\frac{s}{s+1}\right)=0,
$$

for $s=0,1, \ldots$, and $Q^{\prime}$. Then

$$
f_{Q^{\prime} 0}=f_{Q^{\prime} 1}=\cdots=f_{Q^{\prime} Q^{\prime}} .
$$

Proof. We may use Eq. (35) with $s=Q^{\prime}-1$ to determine $f_{Q^{\prime}, Q^{\prime}-1}$ uniquely in terms of $f_{Q^{\prime}, Q^{\prime}}$; the same equation with $s=Q^{\prime}-2$ to determine $f_{Q^{\prime}, Q^{\prime}-2}$ uniquely in terms of $f_{Q^{\prime}, Q^{\prime}}$ and $f_{Q^{\prime}, Q^{\prime}-1} ; \ldots$; and the same equation with $s=0$ to determine $f_{Q^{\prime} 0}$ uniquely in terms of $f_{Q^{\prime} Q^{\prime}}, f_{Q^{\prime}, Q^{\prime}-1}, \ldots$, and $f_{Q^{\prime} 1}$. In other words, $f_{Q^{\prime} 0}, f_{Q^{\prime} 1}, \ldots$, and $f_{Q^{\prime}, Q^{\prime}-1}$ depend on $f_{Q^{\prime} Q^{\prime}}$ uniquely. By Lemma A.1,

$$
f_{Q^{\prime} 0}=f_{Q^{\prime} 1}=\cdots=f_{Q^{\prime} Q^{\prime}}
$$

Q.E.D.

\section{References}

[1] P. Di Francesco, P. Ginsparg, and J. Zinn-Justin, Phys. Rept. 254 (1995) $1-133$ [hep-th/9306153].

[2] J. Ambjørn, B. Durhuus, and T. Jónsson, Quantum Geometry, Cambridge Monographs on Mathematical Physics (Cambridge University Press, 1997).

[3] R. Nakayama, Phys. Lett. B 325 (1994) 347-353 [hep-th/9312158].

[4] Jan Ambjørn and Renate Loll, Nuclear Physics B 536 (1999) 407-434 [hep-th/9805108].

[5] P. Di Francesco, E. Guitter, and C. Kristjansen, Nucl. Phys. B 567 (2000) 515 - 553 [hep-th/9907084].

[6] P. Di Francesco, E. Guitter, and C. Kristjansen, Nucl. Phys. B 608 (2001) 485 - 526 [hep-th/0010259].

[7] B. Durhuus and C.-W. H. Lee, Nuclear Physics B 623 (2002) 201-219 [hep-th/0108149].

[8] C.-W. H. Lee and R. B. Mann, Nucl. Phys. B 675 (2003) 139-158 [hep-th/0307231].

[9] C.-W. H. Lee and S. G. Rajeev, Int. J. Mod. Phys. A 14 (1999) 4395-4456 [hep-th/9906060].

[10] C.-W. H. Lee and S. G. Rajeev, Phys. Lett. B 436 (1998) 91—96 [hep-th/9806019]. 